\title{
Circulating low density neutrophils of breast cancer patients are associated with their worse prognosis due to the impairment of T cell responses
}

\author{
Diana P. Saraiva ${ }^{1, *}$, Bruna F. Correia ${ }^{1, *}$, Rute Salvador $^{1}$, Nídia de Sousa1, António \\ Jacinto ${ }^{1}$, Sofia Braga ${ }^{1,2}$ and M. Guadalupe Cabral ${ }^{1}$ \\ ${ }^{1}$ iNOVA4Health, CEDOC, NOVA Medical School (NMS), Universidade Nova de Lisboa, Lisbon, Portugal \\ ${ }^{2}$ Instituto CUF de Oncologia, Lisbon, Portugal \\ *These authors contributed equally to this work \\ Correspondence to: M. Guadalupe Cabral, email: guadalupe.cabral@nms.unl.pt \\ Keywords: breast cancer; chemotherapy response; tumor-associated neutrophils; low density neutrophils; biomarker \\ Received: July 29, $2021 \quad$ Accepted: November 10, $2021 \quad$ Published: November 23, 2021
}

Copyright: ( 2021 Saraiva et al. This is an open access article distributed under the terms of the Creative Commons Attribution License (CC BY 3.0), which permits unrestricted use, distribution, and reproduction in any medium, provided the original author and source are credited.

\section{ABSTRACT}

Neutrophils are prominent immune components of tumors, having either antitumor (N1) or pro-tumor activity (N2). Circulating neutrophils, divided into high density neutrophils (HDN) and low density neutrophils (LDN), functionally mirror those $\mathrm{N} 1$ and $\mathrm{N} 2$ cells, respectively. LDN are rare in non-pathological conditions, but frequent in cancer, exhibiting a pro-tumor phenotype. These findings have been mainly demonstrated in animal models, thus proper validation in humans is still imperative. Here, we observed that LDN were increased in the blood of breast cancer (BC) patients, particularly with metastatic disease. Within the population of non-metastatic patients, LDN were more prevalent in patients with poor response to neoadjuvant chemotherapy than patients with a good response. The higher incidence of LDN in BC patients with severe disease or resistance to treatment can be explained by their pro-tumor/immunosuppressive characteristics. Moreover, the percentage of LDN in BC patients' blood was negatively correlated with activated cytotoxic $T$ lymphocytes and positively correlated with immunosuppressive regulatory $\mathrm{T}$ cells. The ability of LDN to spoil anti-tumor immune responses was further demonstrated ex vivo. Hence, this study reveals the potential of LDN as a biomarker of BC response to treatment and opens new avenues for developing new immunotherapies.

\section{INTRODUCTION}

Breast cancer $(\mathrm{BC})$ is the main type of cancer in women worldwide, with more than 2 million new cases per year [1]. Additionally, it is the main cause of cancer-related deaths in women, accounting for more than 600000 deaths annually [1]. BC can be molecularly divided into three different subtypes, namely tumors with an overexpression of the estrogen receptor $(\mathrm{ER}+)$, which can concomitantly increase the progesterone receptor (PR); tumors with amplification on the HER2 (human epidermal growth factor receptor 2) gene; and tumors that lack the three above mentioned markers and are classified as Triple Negative Breast Cancer (TNBC).
Tumor development and response to treatment are highly dependent on the interactions that occur in the tumor immune microenvironment (TIME). Actually, the presence of tumor-infiltrating lymphocytes (TILs) has been correlated with improved pathological complete response (pCR) following neoadjuvant chemotherapy (NACT) in BC patients [2]. Additionally, the neutrophilto-lymphocyte ratio (NLR) assessed in peripheral blood has also been correlated with BC response to NACT, since most studies advocate that a high NLR is predictive of a poor response to treatment [3]. However, data is still conflicting, and other authors claim no association between the NLR and prognosis, implying that the use of the NLR in a clinical setting must be extensively studied first $[4,5]$. In fact, tumors have complex mechanisms to 
escape immune surveillance [6] and, in addition, both lymphocytes and neutrophils are composed of different cell subtypes with opposite functions (anti- and pro-tumor), which can explain why these markers of $\mathrm{BC}$ response to treatment are still scarcely used in a clinical routine.

Tumor-associated neutrophils (TANs) can be divided into two different populations - N1 and N2, similar to the macrophage's polarization. N1 are pro-inflammatory and anti-tumor, with the capacity to stimulate effector $\mathrm{T}$ lymphocytes to eliminate tumor cells; while N2 have pro-tumor, immunosuppressive and angiogenic features [7]. The presence of transforming growth factor- $\beta$ (TGF- $\beta$ ) in the TIME was found to be necessary for the transformation of TANs to an $\mathrm{N} 2$ phenotype $[7,8]$. Mirroring the polarization of TANs, circulating neutrophils can also be divided into two subpopulations - high density neutrophils (HDN) and low density neutrophils (LDN), distinguished based on their density gradient, which are phenotypically similar to $\mathrm{N} 1$ and N2, respectively. Although HDN and LDN share common markers, namely CD11b, CD66b, CD15, their expression is higher in LDN [9-11]. LDN are absent in healthy individuals' blood, emerging only during inflammatory and/or pathological conditions [12]. LDN are seen as a mixture of both mature and immature neutrophils, with altered functions and immunosuppressive properties, such as the stimulation of regulatory $\mathrm{T}$ cells (Tregs), the release of reactive oxygen species (ROS), nitric oxide and arginase, which will inhibit effector CD8+ T lymphocytes' activity [13, 14]. Besides these mechanisms, immunosuppressive neutrophils were shown to express the programmed cell death ligand 1 (PD-L1, [15]), which is an immune checkpoint that hampers effector T lymphocytes' response, when bound to its receptor PD-1. The role of LDN in cancer have been intensively studied in the past years, mainly in animal models, and it was observed that this subset of neutrophils also has the capacity to promote metastization of cancer cells [16], due, in part, to its extraordinary ability to release neutrophil extracellular traps (NETs) to the environment, which can entrap circulating tumor cells and help in their migration towards secondary niches [16].

Yet, studies in this topic using human samples are scarce, and considering that human and mice neutrophils exhibit significant biological differences, efforts to validate in cancer patients the results assessed in animal models regarding LDN are crucial to fully comprehend their future clinical utility.

Thus, here we evaluated the impact of circulating LDN in BC patients, corroborating that a high frequency of these cells is associated with a poor prognosis. More specifically, our results suggested that in non-metastatic $\mathrm{BC}, \mathrm{LDN}$ can predict the response to NACT, as patients with poor response to this treatment showed an increased percentage of LDN pre-treatment. Additionally, we performed a thorough phenotypic and functional characterization of these $\mathrm{BC}$ patient-derived LDN in comparison to HDN and demonstrated that LDN are highly activated neutrophils; with increased capacity to phagocyte bacteria, produce ROS and form NETs; and with an immunosuppressive action towards CD4+ and CD8+ T lymphocytes, which may justify our clinical observations.

\section{RESULTS}

\section{LDN are associated with a worse prognosis of breast cancer patients, in particular, with a poor response to neoadjuvant chemotherapy}

48 non-metastatic and 12 metastatic breast cancer (BC) patients were enrolled in this study. The main characteristics of these patients are described in Table 1. All non-metastatic patients were treated with neoadjuvant chemotherapy (NACT), having received no prior treatment. The median age of the non-metastatic patients was 57 and the median body mass index was 26 . The majority of these patients already had the disease extended to the axillary lymph nodes. Metastatic patients, with a median age of 64 were treated with adjuvant chemotherapy and in some cases hormone therapy or trastuzumab (the anti-HER2 monoclonal antibody), depending on the BC subtype.

Using blood samples from these BC patients, we determined the frequency of low density neutrophils (LDN) and the frequency of high density neutrophils (HDN), after density gradient centrifugation. We also investigated the presence of LDN in healthy donors. As expected, we observed that LDN are almost absent in healthy individuals when compared to non-metastatic and metastatic BC patients ( $p<0.01$, Figure 1A). Within $\mathrm{BC}$ patients, we also observed a significantly higher percentage of LDN in metastatic patients than in nonmetastatic patients ( $p<0.05$, Figure $1 \mathrm{~A})$, corroborating the idea that LDN are more frequent in more advanced, metastatic patients, as it occurred in mice models [16, 17].

Then, we further investigated the impact of LDN in the response to standard treatment, particularly in non-metastatic BC patients selected for NACT, since a poor response to this treatment is a predictive factor for recurrence and disease progression $[18,19]$. NACT is the treatment of choice for BC patients with tumors larger than $2 \mathrm{~cm}$ and/or with disease extended to axillary lymph nodes, or with inflammatory and inoperable tumors, independently of the BC subtype. After six months of treatment, the response is assessed by evaluating the remaining tumor after surgery. Patient response was classified according to the pathological and clinical criteria already established [20]. Briefly, NACT-responders were classified as patients that achieved a pathological complete response $(n=10)$ or that had a significant down-staging without axillary lymph node involvement following NACT $(n=9)$. NACT non-responders were classified as patients that did not achieve a tumor down-staging and/ 
Table 1: Characteristics of patients enrolled in the study - blood donors. Clinical data, such as subtype of breast cancer, tumor dimension, Ki67 (related to the tumor proliferation rate), node status and treatment are also summarized

\begin{tabular}{|c|c|c|c|}
\hline & \multicolumn{2}{|c|}{ Patients } & \multirow{2}{*}{ Healthy Donors } \\
\hline & Non-metastatic & Metastatic & \\
\hline Number of Subjects & 48 & 12 & 7 \\
\hline Age & $\begin{array}{c}\text { Median: } 57 \\
\text { (Range: } 31-80 \text { ) }\end{array}$ & $\begin{array}{l}\text { Median: } 64 \\
\text { (range: } 30-87 \text { ) }\end{array}$ & range: $18-65$ \\
\hline Body Mass Index (BMI) & $\begin{array}{c}\text { Median: } 26.03 \\
\text { (range: } 17.22-46.68 \text { ) }\end{array}$ & $\begin{array}{c}\text { Median: } 25.05 \\
\text { (range: } 18.03-35.56 \text { ) }\end{array}$ & NA \\
\hline $\mathbf{E R}+(\mathbf{P R}-/+)$ & $29.27 \%$ & NA & NA \\
\hline $\begin{array}{l}\text { HER2+ including triple } \\
\text { positive breast cancer }\end{array}$ & $51.22 \%$ & NA & NA \\
\hline TNBC & $19.51 \%$ & NA & NA \\
\hline Dimension (mm) & $\begin{array}{l}\text { Median: } 34 \\
\text { (range: 6-110) }\end{array}$ & NA & NA \\
\hline Ki67 & $\begin{array}{l}\text { Median: 35\% } \\
\text { (range: 5-98\%) }\end{array}$ & NA & NA \\
\hline Axillary lymph node & Positive $-63.64 \%$ & NA & NA \\
\hline invasion status & Negative $-36.36 \%$ & NA & NA \\
\hline \multicolumn{4}{|l|}{ Treatment } \\
\hline NACT & $100 \%$ & NA & NA \\
\hline NACT response & $52.78 \%$ & & \\
\hline NACT non-response & $47.22 \%$ & & \\
\hline Adjuvant chemotherapy + Tamoxifen & NA & $72.73 \%$ & NA \\
\hline Adjuvant chemotherapy + Trastuzumab & NA & $27.27 \%$ & NA \\
\hline \multicolumn{4}{|l|}{ Metastasis location } \\
\hline Bone & NA & 5 in 12 & NA \\
\hline Bone marrow & NA & 1 in 12 & NA \\
\hline Liver & NA & 2 in 12 & NA \\
\hline Lungs & NA & 4 in 12 & NA \\
\hline Lung pleura & NA & 2 in 12 & NA \\
\hline
\end{tabular}

"NA: non-applicable.

or that still had disease extension to the axillary lymph nodes after NACT $(n=17)$. Remarkably, we observed that NACT non-responders had a higher percentage of LDN, before starting the treatment, when compared to NACT responders $(p<0.05$, Figure 1B), thus suggesting this immune trace as a probable predictive biomarker.

Since NACT is administered to BC patients independently of the subtype, we compared the levels of LDN in the blood of ER+, HER2 and TNBC patients, to see whether the higher prevalence of LDN is associated with a particular BC subtype. Curiously, ER+ BC patients had a significantly higher percentage of LDN, when compared to HER2 ( $p=0.0007$, Figure 1C) and TNBC ( $p$ $=0.0451$, Figure $1 \mathrm{C}$ ), highlighting that the assessment of LDN could be a crucial tool in determining, in advance, the response to treatment, especially for $\mathrm{ER}+$ patients.

Total blood neutrophils have already been implied in the prediction of $\mathrm{BC}$ response to NACT, with the neutrophil-to-lymphocyte (NLR) ratio [21, 22]. However, data on the predictive power of NLR are still conflicting, for instance, different studies suggest distinct threshold values. To have an idea of the performance of the percentage of LDN as a predictive biomarker in comparison with the NLR in this BC cohort, we assessed the NLR taking into account the percentage of total neutrophils and lymphocytes in the whole blood of non-metastatic patients (Supplementary Figure 1A). The NLR was then calculated, and the BC cohort divided into NACT-responders and non-responders, according to the clinical information (Supplementary Figure 1B). We observed no differences in the NLR when comparing both groups (Supplementary Figure 1B), suggesting that the determination of LDN may be more useful than NLR to predict, in advance, the likelihood of a patient to respond to NACT.

Additionally, we also used fresh biopsies and surgical specimens of $\mathrm{BC}$ patients selected either for NACT or surgery following adjuvant chemotherapy and assessed the percentage of tumor-associated neutrophils (TANs). The main characteristics of these patients 
are described in Table 2. The median value of TANs percentage was obtained, and the patients were then divided into two groups: patients with TANs below or above the median value. We performed a follow-up of these patients for 34 months and the progression-free survival (PFS) was determined (Supplementary Figure $2)$. Interestingly, the PFS was inferior in patients with a higher percentage of TANs $(p=0.015$, hazard ratio $=4.35$ (95\% CI 1.46-19.62), Supplementary Figure 2) when compared to patients with a lower percentage of TANs (hazard ratio $=0.23$ (95\% CI 0.05-0.69), Supplementary Figure 2). However, due to the lack of matched blood and tumor samples, it was not possible to establish a correlation between the frequency of TANs and LDN. Nevertheless, the obtained results show that patients with higher percentage of TANs start to progress earlier and/or have a lower PFS when compared to $\mathrm{BC}$ patients with lower percentage of
TANs. This result further demonstrates the importance of neutrophils in BC.

\section{LDN are highly activated neutrophils with increased neutrophil-associated activities}

To better understand why LDN are associated with worse BC prognosis, we characterized phenotypically and functionally the LDN and HDN subsets. In particular, we assessed the percentage and the median fluorescence intensity (MFI) of CD11b, CD66b, CD33 and PD-L1. CD11b and CD66b are adhesion molecules expressed on activated neutrophils; CD33 is a marker of neutrophils' maturation status, since its expression decreases with cell maturation; PD-L1 is an inhibitory immune checkpoint, capable of decreasing effector immune responses through the impairment of T lymphocytes' activity. The percentage of these markers was similar between LDN and HDN,
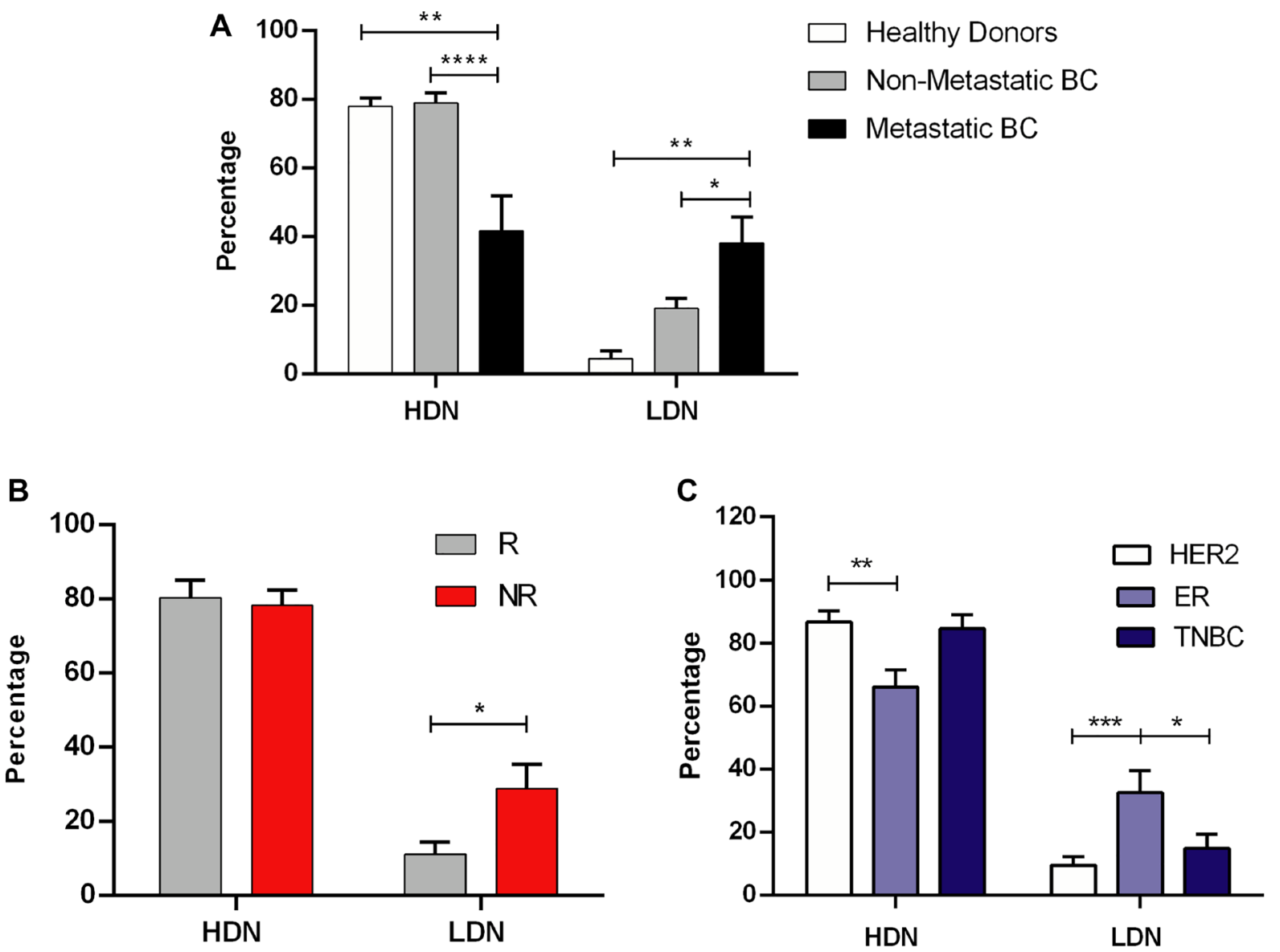

Figure 1: Low density neutrophils are associated with poor response to neoadjuvant chemotherapy and with advanced stages of breast cancer. (A) Percentage of high density neutrophils (HDN) and low density neutrophils (LDN) in the whole blood of healthy donors (white bars, $n=7$ ), in non-metastatic breast cancer (BC) patients (grey bars, $n=48$ ) and metastatic BC patients (black bars, $n=12$ ). (B) Percentage of high density neutrophils (HDN) and low density neutrophils (LDN) in the whole blood of non-metastatic $\mathrm{BC}$ patients with response to NACT (R, grey bars, $n=19)$ and without response to NACT (NR, red bars, $n=17)$. (C) Percentage of high density neutrophils (HDN) and low density neutrophils (LDN) in the whole blood of HER2 non-metastatic BC patients (white bars, $n=20)$, of estrogen receptor (ER+) (light blue bars, $n=15$ ) and in triple negative non-metastatic BC patients (TNBC, dark blue bars, $n=10)$. Data are represented as mean \pm SEM. Statistical analysis: two-way ANOVA with Tukey's multiple comparisons (A) or with Sidak's multiple comparisons (B and C), ${ }^{*} p<0.05,{ }^{* *} p<0.01,{ }^{* * *} p<0.001,{ }^{* * * *} p<0.0001$. 
Table 2: Characteristics of patients enrolled in the study - biopsy and/or tumor donors. Clinical data, such as subtype of breast cancer, tumor dimension, Ki67 (related to the tumor proliferation rate), node status and treatment are also summarized

\begin{tabular}{lc}
\hline Number of Subjects & Non-metastatic Patients \\
Age & 97 \\
Body Mass Index (BMI) & Median: 60 (range: $35-87$ ) \\
ER+ (PR -/+) & Median: 26.09 (range: 17.65-46.64) \\
HER2+ including triple positive breast cancer & $64.29 \%$ \\
TNBC & $23.47 \%$ \\
Dimension (mm) & $12.24 \%$ \\
Ki67 & Median: 23 (range: $7-88)$ \\
Axillary lymph node & Median: 20\% (range: $2-82 \%)$ \\
invasion status & Positive - $43.01 \%$ \\
Treatment & Negative - 56.99\% \\
$\quad$ NACT & \\
$\quad$ Other chemotherapeutic treatments & $33 \%$ \\
\hline
\end{tabular}

except for PD-L1 which was significantly higher in LDN $(p=0.03$, Figure $2 \mathrm{~A})$, suggesting that $\mathrm{LDN}$ can have an augmented immunosuppressive action. When considering the MFI, both CD11b and CD66b are elevated in $\operatorname{LDN}(p$ $<0.01$, Figure $2 \mathrm{~B}$ ), meaning that these markers are more expressed in LDN, highlighting their superior activated state when compared to HDN.

We performed the immunophenotyping analysis of HDN and LDN in both metastatic and non-metastatic BC patients (Supplementary Figure 3) and observed that the percentage of PD-L1 is increased in $\mathrm{HDN}(p<$ 0.05 , Supplementary Figure $3 \mathrm{~A})$ and in $\operatorname{LDN}(p<0.01$, Supplementary Figure 3C) of metastatic patients when compared to non-metastatic. Nevertheless, LDN of metastatic $\mathrm{BC}$ patients still have a higher percentage of PD-L1, when compared to HDN (data not shown). On the other hand, the expression level of CD11b was lower in HDN $(p<0.001$, Supplementary Figure 3B) and in LDN $(p<0.05$, Supplementary Figure 3D) of metastatic patients when compared to non-metastatic. These results imply that the neutrophils of metastatic patients have an even more pronounced immunosuppressive phenotype.

Besides assessing the expression of these markers, we analyzed the neutrophils' function in both subsets. This function relies upon three main distinct activities: the capacity to phagocyte bacteria or other pathogens, the ability to generate an oxidative burst and finally the release of extracellular traps (NETs) through a process called NETosis.

To evaluate the phagocytic capacity, we used FITClabelled E. coli and incubated these bacteria with both $\mathrm{HDN}$ and LDN at $37^{\circ} \mathrm{C}$ and $4^{\circ} \mathrm{C}$ (as a negative control). By flow cytometry, we observed that LDN had a higher FITC internalization $(p<0.0001$, Figure 2C) than HDN, which is correlated with a higher amount of bacteria phagocytosed.
The oxidative burst was assessed by quantifying the production of reactive oxygen species (ROS) using the DCFH2-DA probe, following neutrophils' stimulation with PMA. When stimulated, both neutrophils' subpopulations had an identical capacity to produce ROS (Figure 2D), which was significantly higher than their unstimulated counterparts $(p=0.0005$ and $p=0.01$ for HDN and LDN, respectively, Figure 2D). Though, interestingly, LDN tended to have higher ROS production without any stimulation when compared to HDN also unstimulated (Figure 2D).

NETs are composed by decondensed chromatin, histones, cytoplasmic proteins and granular enzymes, such as myeloperoxidase (MPO) and neutrophil elastase, that attach to the DNA [23]. In a tumor context, NETs have been shown, mainly using mice models, to entrap malignant cells, supporting the early adhesion of circulating tumor cells in distant organ sites [24, 25]. We analyzed the formation of these structures in both HDN and LDN with or without PMA stimulation. Both subtypes were able to release NETs (Figure 2E and 2F) and, although HDN showed NETs that occupied a larger area when stimulated (compared to stimulated LDN, $p=0.002$, Supplementary Figure 4A), MPO was significantly reduced in stimulated HDN (compared to stimulated LDN, $p=0.04$, Figure 2E). Interestingly, nonstimulated LDN were able to form NETs with a higher area $(p=0.06$, Supplementary Figure 4A) and MPO intensity ( $p=0.03$, Figure $2 \mathrm{E}$ ) when compared to HDN also unstimulated, demonstrating their hardwired capacity to release these structures, even without further stimulus. Besides assessing NETs' area and MPO intensity, we also quantified the nuclear area, since nuclear enlargement is an initial step of NETosis. Again, unstimulated LDN have an increased nuclei area when compared to unstimulated 

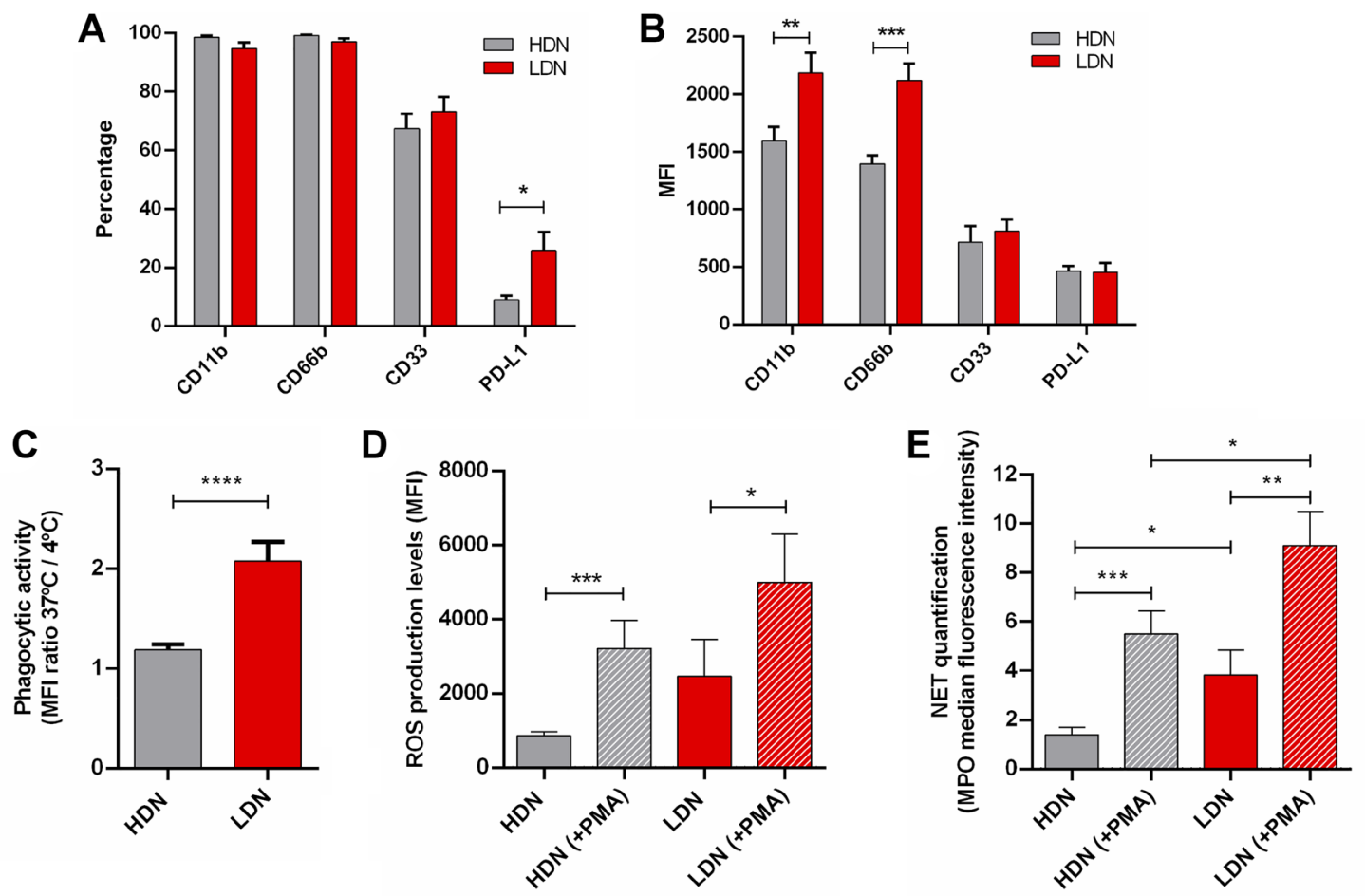

$\mathbf{F}$

HDN
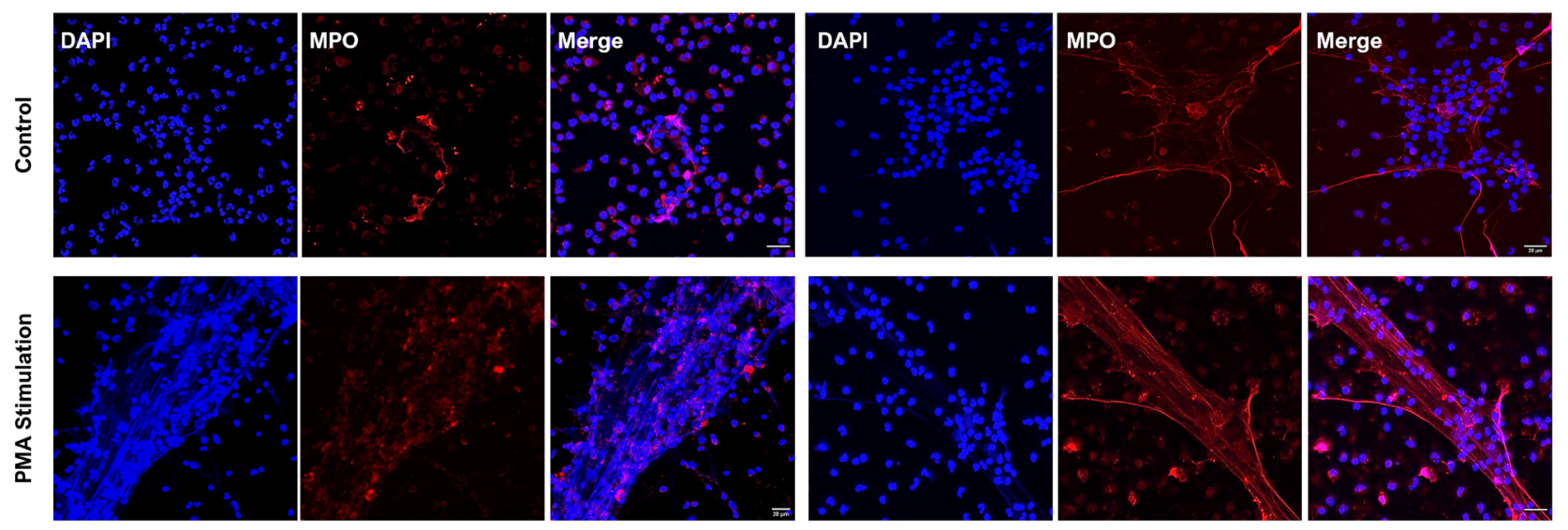

Figure 2: Low density neutrophils are more activated, have a higher phagocytic capacity, produce more reactive oxygen species and neutrophil extracellular traps than high density neutrophils. (A) Percentage of high density neutrophils (HDN, grey bars) and low density neutrophils (LDN, red bars) positive for CD11b, CD66b, CD33 and PD-L1 (assessed by flow cytometry). (B) Median fluorescence intensity (MFI) of CD11b, CD66b, CD33 and PD-L1 present in high density neutrophils (HDN, grey bars) and low density neutrophils (LDN, red bars). Data in (A) and (B) correspond to 48 non-metastatic breast cancer patients. (C) Phagocytic capacity analyzed as the ratio between the median fluorescence intensity (MFI) of FITC-labelled $E$. coli incubated with neutrophils at $37^{\circ} \mathrm{C}$ and $4^{\circ} \mathrm{C}$ in high density neutrophils (HDN, grey bar, $n=11$ ) and low density neutrophils (LDN, red bar, $n=12$ ). (D) Levels of released reactive oxygen species (ROS) assessed by flow cytometry of high density neutrophils without stimulation (HDN, grey bar, $n=14$ ) and with PMA stimulation (HDN (+PMA), grey bar with stripes, $n=14$ ), of low density neutrophils without stimulation (LDN, red bar, $n=$ 14) and with PMA stimulation (LDN (+PMA), red bar with stripes, $n=14)$. (E) Neutrophil extracellular traps (NETs) quantification by the median fluorescence intensity of myeloperoxidase (MPO) in high density neutrophils without stimulation (HDN, grey bar, $n=11$ ) and with PMA stimulation (HDN (+PMA), grey bar with stripes, $n=11$ ), of low density neutrophils without stimulation (LDN, red bar, $n=11$ ) and with PMA stimulation (LDN (+PMA), red bar with stripes, $n=11$ ). Each $\mathrm{n}$ represents the mean value of 3 different images per patient. (F) Images of neutrophil extracellular traps (NETs) in high and low density neutrophils with and without PMA stimulation analyzed with DNA staining (DAPI, blue channel) and MPO staining in red. Both channels were merged and the $Z$ stacks ( $1 \mu \mathrm{m}$ between each stack) were projected with the maximum fluorescence intensity. Scale bar (white line): $20 \mu \mathrm{m}$. Data in A-E are represented as mean \pm SEM. Statistical analysis: two-way ANOVA with Sidak's multiple comparisons (A and B) and Mann-Whitney (C-E) tests, ${ }^{*} p<0.05,{ }^{* * *} p<0.01,{ }^{* * *} p<0.001$, ${ }^{* * * *} p<0.0001$. 
HDN ( $p=0.003$, Supplementary Figure 4B), even if this difference was abrogated when neutrophils were PMAstimulated (Supplementary Figure 4B).

The increased capacity of LDN to phagocyte bacteria, release ROS and form MPO-containing NETs, even in the absence of stimulation, appear to be correlated with the fact that this subtype has a higher activation level, seen by the increased expression of CD11b and CD66b. These features are associated with augmented antimicrobial responses, but there is also growing evidence for these increased activities being implicated in the acceleration of tumor progression [26].

\section{LDN are correlated with immunosuppressive molecules and regulatory $\mathrm{T}$ lymphocytes}

We analyzed the level of several cytokines in the plasma of BC patients, namely IL-8, IL-10, IL-17, IFN- $\gamma$, TGF- $\beta$ and CCL17. The chemokine CCL17 is produced by neutrophils and previous studies have shown that its expression is increased in pro-tumor N2 TANs [8]. Accordingly, a positive correlation between the level of LDN and the concentration of CCL17 in BC patients' plasma ( $r=0.57, p=0.0007$, Figure 3A, Supplementary Table 1) was found. CCL17 is the ligand of CCR4, expressed in $\mathrm{T}$ lymphocytes, especially in regulatory $\mathrm{T}$ cells (Tregs) and acts as a chemoattractant of these lymphocytes. Consequently, we observed that CCR4+ Tregs were increased in the blood of patients that presented higher levels of LDN, as a positive correlation was also observed between these two populations $(r=0.34, p=$ 0.03 , Figure $3 \mathrm{~B}$ ). We also assessed IL-8, a chemokine known to be involved in neutrophil recruitment. Interestingly, we observed a positive correlation between the level of HDN and the concentration of IL-8 in the patients' plasma ( $r=0.5128, p=0.0208$, Supplementary Table 1). However, the same was not observed for the level of LDN (Supplementary Table 1), which may indicate that this particular subset of neutrophils is not being recruited by IL-8, but by another chemokine, for instance CCL17, as suggested by the results above mentioned. As for IFN- $\gamma$, IL-10 and IL-17, they were chosen because IFN- $\gamma$ reflects the activation status of cytotoxic $\mathrm{T}$ lymphocytes (CTLs), whereas IL-10 reflects an anti-inflammatory environment, and IL-17 is a key player in the promotion of neutrophil expansion and polarization towards an immunosuppressive phenotype. However, the levels of IL-10 and IFN- $\gamma$ did not show any correlation with LDN (Supplementary Table 1), and IL-17 was not detected in patients' plasma (Supplementary Table 1).

Since TGF- $\beta$ has the capacity to transform "normal" neutrophils into neutrophils with a pro-tumor phenotype, we also evaluated this cytokine in the plasma of $\mathrm{BC}$ patients and established a positive correlation between its level and the percentage of LDN in the blood $(r=0.45, p$ $=0.025$, Figure 3C).
Ultimately, it was observed that activated effector CTLs, expressing the activation marker CD69, showed a tendency to be negatively correlated with the percentage of LDN ( $r=-0.38, p=0.053$, Figure 3D) in patients' blood. Thus, it seems that LDN, besides expressing PD-L1, could also indirectly exert immunosuppression towards $\mathrm{T}$ lymphocytes, possibly via CCR4+ Tregs, which in turn contribute to inhibit the activity of CTLs, and consequently anti-tumor responses.

\section{LDN can reduce the activation and proliferation of T lymphocytes}

To confirm the hypothesis that LDN of BC patients impair T lymphocytes' activity, we conducted in vitro experiments to evaluate the impact of this neutrophils' subset in the activation and proliferation of $\mathrm{T}$ lymphocytes. Therefore, we performed a co-culture of LDN with peripheral blood mononuclear cells (PBMCs, depleted of CD15+ cells, which are precisely the neutrophils that appear in PBMCs fraction, upon the density gradient centrifugation) from the same patient (Figure 4A and 4B), with or without stimulation with PMA and ionomycin. As a control, we used a monoculture of PBMCs (also depleted of CD15+ cells). While no significant differences were found in the unstimulated condition, the stimulated PBMCs plus LDN demonstrated an overall reduction in the activation markers - CD25, CD69 and HLA-DR and in the proliferation marker (Ki67) in both CD4+ (Figure 4A) and CD8+ T lymphocytes (Figure 4B), when compared to stimulated PBMCs incubated in the absence of LDN. This observation corroborates the idea that LDN weakens the ability of effector T lymphocytes to become activated upon stimulation.

As an additional readout of this LDN-derived $\mathrm{T}$ lymphocyte suppression, we assessed the levels of secreted IFN- $\gamma$ in the cultures' supernatants (Figure 4C). As expected, IFN- $\gamma$ production was reduced in stimulated T lymphocytes in contact with LDN $(p=0.007$, Figure 4C). Thus, patient derived-LDN have indeed an immunosuppressive phenotype, with the capacity to reduce the activation and the proliferation of effector $\mathrm{T}$ lymphocytes.

Likewise, we also assessed the levels of released CCL17 in cultured HDN and LDN, with or without a 3 hours stimulation with PMA (Figure 4D). LDN without stimulation showed a higher capacity to produce this chemokine when compared to HDN ( $p=0.04$, Figure 4D). When both subtypes were stimulated, this difference was further enhanced ( $p=0.02$, Figure 4D), showcasing the higher capacity of LDN to produce CCL17. This result combined with the results of the previous subsection supports the idea, suggested by animal studies [27], that LDN mediate T lymphocytes impaired immune response. Namely, LDN may recruit CCR4+ Tregs, via the release of CCL17, and downregulate the activation of effector $\mathrm{T}$ lymphocytes. 


\section{DISCUSSION}

Neutrophils have gained significant interest in the past years in the field of tumor immune microenvironment (TIME). These immune cells, which were previously only seen as the first responders to a pathogen, were encountered in the TIME, and their major role in cancer biology is becoming increasingly clear $[12,13]$. The relevance of neutrophils in tumorigenesis was first suggested by the observation that cancer patients, especially the population with more advanced and aggressive disease, exhibited an increased neutrophil-tolymphocyte ratio (NLR) in the peripheral blood $[3,21]$. Additionally, a large-scale meta-analysis of expression signatures revealed tumor-associated neutrophils (TANs)signatures as being associated with poor disease outcomes in several types of solid tumors [28]. Indeed, it has been demonstrated that tumor-mediated signals induce the formation of TANs that support tumor growth and metastization; however, there are also studies showing that TANs can also exhibit an anti-tumorigenic phenotype [26].
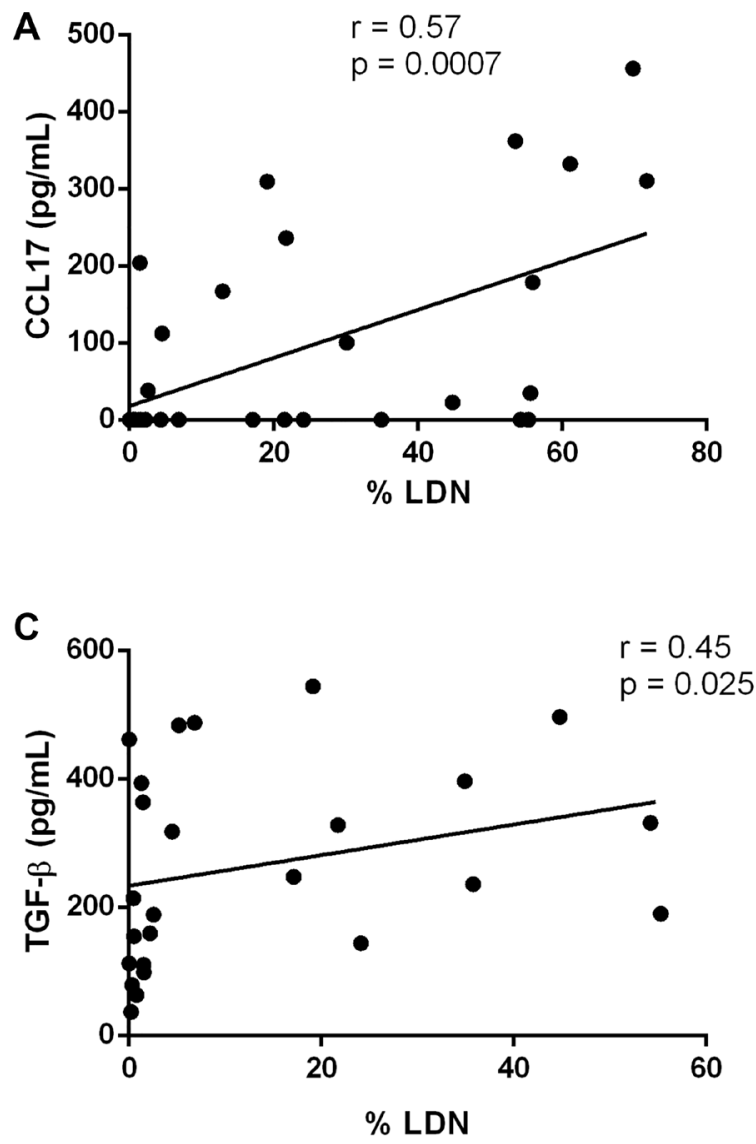

This dichotomy led to the establishment of two different subtypes of TANs - N1 (anti-tumor) and N2 (pro-tumor), which were reflected systemically - high density neutrophils (HDN) and low density neutrophils (LDN), respectively, being the latter mainly present in autoimmune diseases and cancer [29].

The role of the LDN subtype in cancer has been studied particularly in mice models. LDN were shown to have immunosuppressive characteristics and the ability to enhance tumor progression and metastization [16]. Nevertheless, there are various dissimilar aspects of neutrophil biology between humans and mice, and conflicting reports still fuel the debate regarding the role played by human LDN in specific cancers. Hence, studies in human subjects that support these findings are still missing in the field. As such, in a cohort of non-metastatic and metastatic breast cancer $(\mathrm{BC})$ patients, we assess the role of LDN in patients' outcomes, particularly in the response to the conventional neoadjuvant chemotherapy (NACT), their phenotype and function, as well as investigate
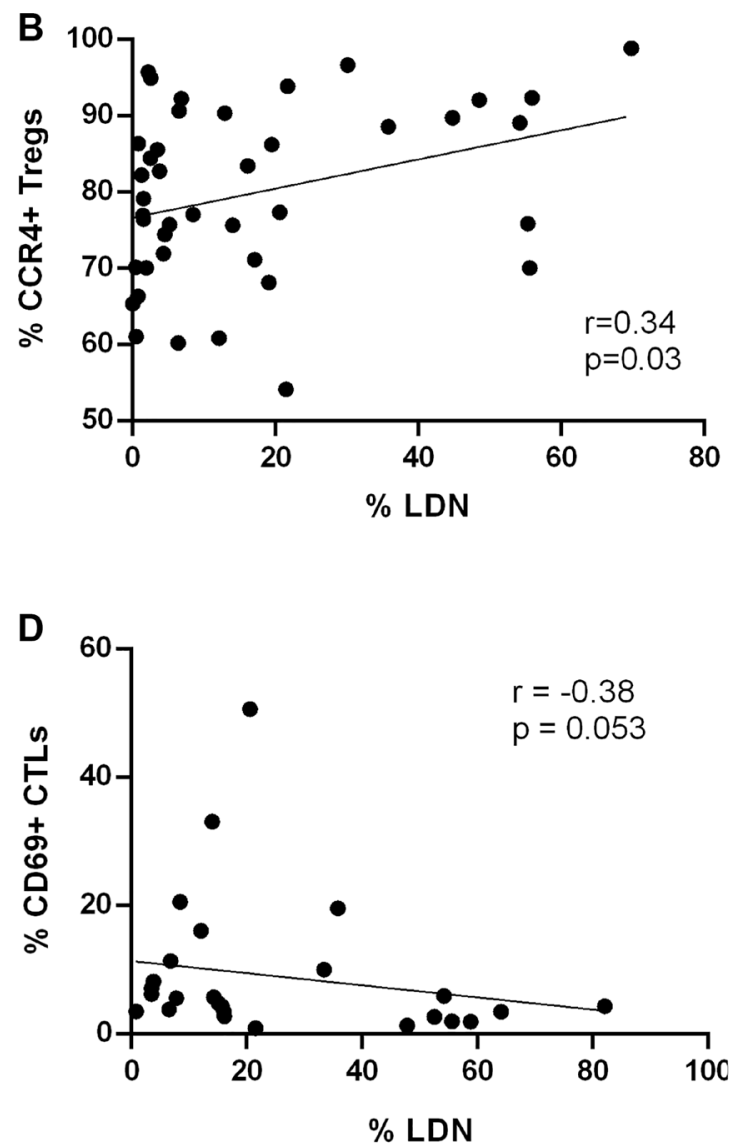

Figure 3: Low density neutrophils are positively correlated with immunosuppressive molecules and regulatory $\mathrm{T}$ cells. (A) Correlation between the percentage of low density neutrophils (LDN) and the concentration of CCL17 in the plasma of breast cancer patients (Spearman $r=0.57, p=0.0007, n=32$ ). (B) Correlation between the percentage of LDN and the percentage of circulating CCR4+ regulatory T cells (Tregs) in breast cancer patients (Spearman $r=0.34, p=0.03, n=41$ ). (C) Correlation between the percentage of low density neutrophils (LDN) and the concentration of TGF- $\beta$ in the plasma of breast cancer patients (Spearman $r=0.45, p=0.025, n=25$ ). (D) Correlation between the percentage of LDN and the percentage of circulating CD69+ cytotoxic T lymphocytes (CTLs) in breast cancer patients (Spearman $r=-0.38, p=0.053, n=27$ ). 
the impact of these human LDN in T lymphocytes' activity.

First, we observed that LDN are absent in the blood of healthy individuals and that metastatic BC patients have a higher percentage of LDN than non-metastatic patients, corroborating the idea that LDN are implicated in metastization $[30,31]$ and accumulate continuously with cancer progression [13]. Additionally, we observed that non-metastatic $\mathrm{BC}$ patients without response to NACT had, before starting the treatment, a significantly higher percentage of LDN when compared to patients with response to NACT. Although a high prevalence of neutrophils in the low density fraction was already observed in cancer patients, including patients with breast and lung carcinomas, most of the patients enrolled in previous studies had advanced disease and no correlation between response to treatment and LDN propagation was established [11, 13].

As such, our observations suggest that the percentage of LDN could potentially be used as a predictive factor to discriminate, prior to treatment, patients that will truly benefit from the treatment and promptly transfer the nonresponders to alternative therapies.

Interestingly, the most studied biomarkers to predict BC response to NACT - TILs and NLR, are usually associated with triple negative breast cancer (TNBC) [2, $32,33]$ and, here, we observed that the subtype of $\mathrm{BC}$ with a higher percentage of LDN was estrogen receptor
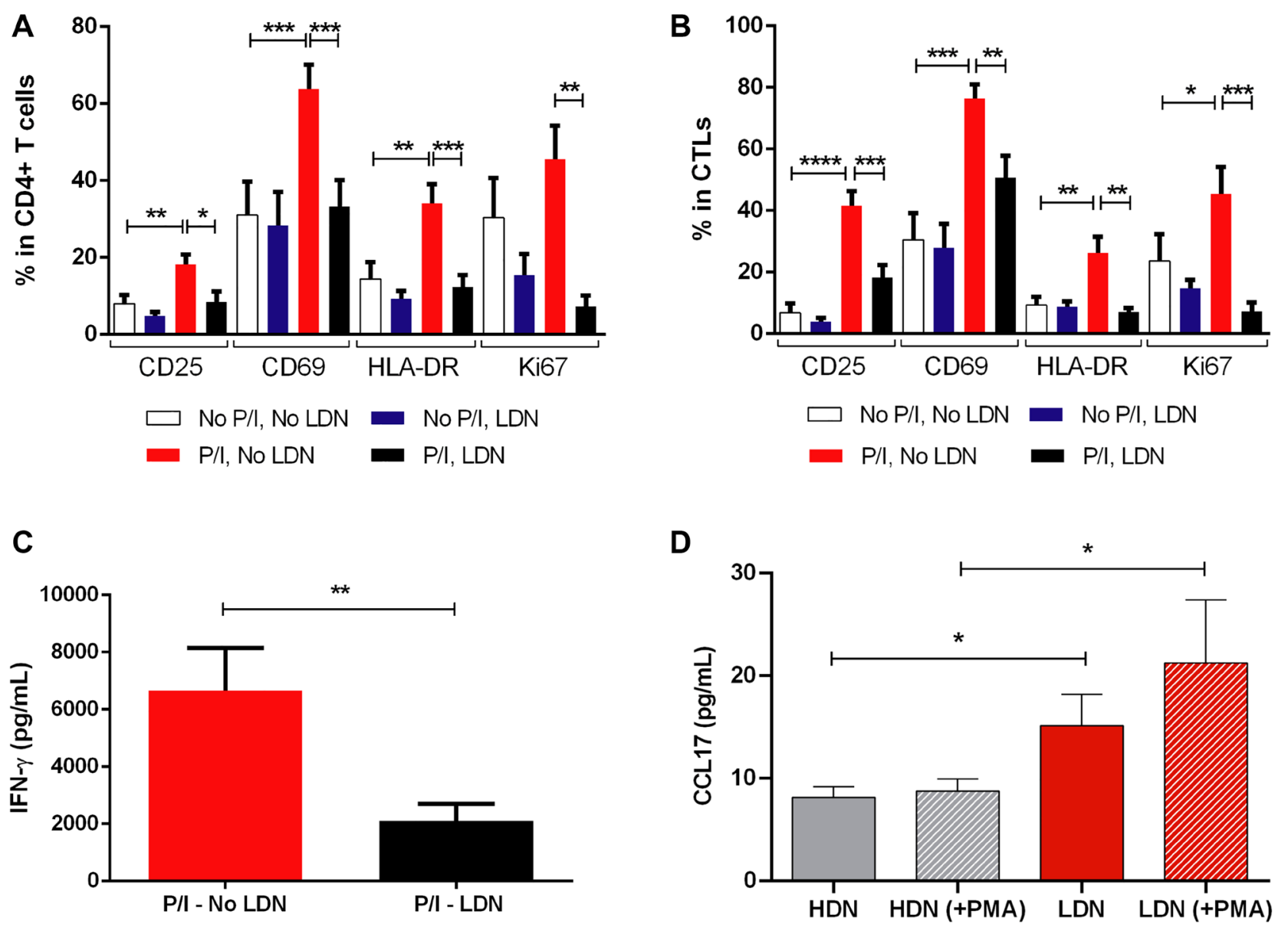

Figure 4: Low density neutrophils are capable of reducing the activation level and the proliferation of effector $\mathrm{T}$ lymphocytes. (A) Percentage of CD25, CD69, HLA-DR and Ki67 in cultured CD4+ T cells without stimulation and without the addition of LDN (No P/I, No LDN, white bars), without stimulation and with the addition of LDN (No P/I, LDN, blue bars), with PMA/ionomycin stimulation and without the addition of LDN (P/I, No LDN, red bars) and with both stimulation and the addition of LDN (P/I, LDN, black bars), $n=10$. (B) Percentage of CD25, CD69, HLA-DR and Ki67 in cultured cytotoxic T lymphocytes (CTLs) without stimulation and without the addition of LDN (No P/I, No LDN, white bars), without stimulation and with the addition of LDN (No P/I, LDN, blue bars), with PMA/ionomycin stimulation and without the addition of LDN (P/I, No LDN, red bars) and with both stimulation and the addition of LDN (P/I, LDN, black bars), $n=10$. (C) Concentration of IFN- $\gamma$ produced in the PBMCs monoculture with PMA/ionomycin stimulation (P/I - No LDN, red bar, $n=8$ ) and in the PBMCs and LDN co-culture with PMA/ionomycin stimulation (P/I - LDN, black bar, $n=8)$. (D) CCL17 produced by cultured high density neutrophils without stimulation (HDN, grey bar) and with PMA stimulation (HDN (+PMA), grey bar with stripes), low density neutrophils without stimulation (LDN, red bar) and with PMA stimulation (LDN (+PMA), red bar with stripes), $n=6$. Data are represented as mean \pm SEM. Statistical analysis: Mann-Whitney (C and D) and $t$-test (A and B), ${ }^{*} p<0.05,{ }^{* *} p<$ $0.01,{ }^{* * *} p<0.001,{ }^{* * * *} p<0.0001$. 
$(\mathrm{ER}+)$. LDN appear as particularly important to predict, in advance, the response to treatment of $\mathrm{BC}$ patients with $\mathrm{ER}+$ tumors, which curiously are normally seen as the $\mathrm{BC}$ patients with better response to treatment and overall survival.

Also, we believe that LDN could be an interesting, alternative biomarker to NLR, with several advantages. Indeed, besides the fact that we did not observe significant differences regarding NLR between NACT-responders and non-responders in our cohort, it is important to note that NLR includes several subsets of cells, which may have different roles in cancer. Yet, it is not completely clear whether NLR is truly representative of pro-tumor neutrophils or simply reflects a tumor associatedinflammatory condition.

Phenotypically, we observed that LDN had a higher percentage of PD-L1 and a higher expression level (MFI) of CD11b and CD66b, when compared to HDN, in accordance with previously published studies [9-11, 34]. These results highlight, on one hand, the greater immunosuppressive status and, on the other, the more activated state of LDN when compared to HDN. Then, we characterized the normal neutrophils' function in both subsets. Interestingly, LDN had a higher capacity to phagocyte FITC-labelled E. coli and to release reactive oxygen species (ROS), which could correlate with the more activated phenotype $\left(\mathrm{CD} 11 \mathrm{~b}^{\text {high }} / \mathrm{CD} 66 \mathrm{~b}^{\text {high }}\right)$. This phenotype of LDN, aligned with what has been mainly reported for animal studies, may contribute to a more aggressive and resistant to treatment BC. Namely, PD-L1 is a well-studied immune checkpoint inhibitor that spoils the anti-tumor immune responses [35]. Additionally, it is known that ROS may contribute to initiate cancer angiogenesis, metastasis and the activation of cell survival signals [36]. The release of ROS was also shown to reduce the function of effector T lymphocytes [13], which could be another way by which LDN impair T lymphocytes' antitumor function. There is also a growing body of evidence for neutrophil activation driving tumor progression and metastasis through a number of pathways [37].

The formation of neutrophil extracellular traps (NETs), also known as the process of NETosis, is a stepwise cascade, starting with the production of ROS, followed by chromatin decondensation and release of DNA, histones and cytoplasmic enzymes (including neutrophil elastase and myeloperoxidase - MPO) to the extracellular space [38]. We observed that both subtypes were capable to form these structures, although MPO intensity was higher in LDN, whereas the NETs' area was increased in HDN. MPO was shown to be added to the NETs' structure in later stages [38], which could suggest that LDN have more mature NETs, although not as large as in HDN. Additionally, MPO present in NETs has been shown to limit $\mathrm{T}$ lymphocytes' activity [39, 40], which again sustains the immunosuppressive role of LDN.
Focusing on LDN, we observed that this neutrophils' subset was positively correlated with the concentration of TGF- $\beta$ and CCL17. TGF- $\beta$ is a known inducer of LDN polarization [7] and is also essential in the process of epithelial-to-mesenchymal transition [41], which culminates in the extravasation of tumor cells from the primary tumor. Moreover, this cytokine is correlated with increased tumor growth [42] and M2 polarization [43], assisting in the maintenance of an immunosuppressive microenvironment. CCL17 is capable of recruiting lymphocytes that express its receptor - CCR4. We observed a positive correlation between LDN and CCR4+ Tregs, suggesting that CCL17-producing LDN can recruit immunosuppressive Tregs (Figure 5). Tregs, on their hand, can inhibit the effector function of CTLs and, accordingly, we observed a tendency for a negative correlation between LDN and activated CTLs (Figure 5).

To better elucidate this issue, we performed ex vivo co-culture assays consisting of PBMCs (depleted of the neutrophils that appear in this mononuclear cells' fraction, upon centrifugation) in the presence or absence of LDN isolated from the same patient. We observed that the T lymphocytes' activation and proliferation that were increased following PMA/ ionomycin stimulation, were significantly reduced in the presence of LDN, attesting the immunosuppressive action of this neutrophil subset.

We have previously reported that activated CTLs (expressing the activation marker HLA-DR) were mainly present in biopsies of $\mathrm{BC}$ patients with good response to NACT and that this biomarker could predict efficiently $\mathrm{BC}$ response to treatment [20]. Combining with the results shown in this study, there seems to be an effect of low density neutrophils in activated $\mathrm{T}$ lymphocytes. This effect is not only indirect, by the recruitment and stimulation of Tregs, but also direct since LDN can decrease the activation and proliferation of effector CTLs.

This study demonstrates that LDN have an important role in breast cancer progression, as well as the potential to be used as a predictive marker of response to NACT. Nevertheless, further studies need to be conducted to validate this marker. Additionally, due to the immunosuppressive action of LDN in effector $\mathrm{T}$ lymphocytes, new targeted immunotherapies could be developed, to inhibit LDN activity and, consequently, release its inhibitory effect on effector cytotoxic T lymphocytes. The manipulation of TGF- $\beta$ or the enhancement of IFN- $\gamma$ activity, have shown to favor neutrophil anti-tumor functions rather than protumor [44]. However, these therapies were proved to be toxic and not well tolerated. Therefore, LDN-target immunotherapies that could assist in the treatment of breast cancer patients with poor response to standard chemotherapy still need to be developed. Although further studies are needed, our results suggest that CCL17 may be a potential therapeutic target. 


\section{MATERIALS AND METHODS}

\section{Patients'samples}

Samples from 157 breast cancer (BC) patients were collected for this study (see the flowchart in Supplementary Figure 5). From the 157 samples, 60 were blood samples (48 non-metastatic BC and 12 metastatic $\mathrm{BC})$; these samples were collected in Vacutainer EDTA tubes (BD Biosciences). Whole blood from 7 healthy donors was collected for comparison studies. Additionally, fresh biopsies and surgical specimens from 97 nonmatched non-metastatic patients were collected in Transfix (Cytomark). Patients' characteristics are described in Tables 1 and 2. These samples were handled one day post-collection and were provided by Hospital de Vila Franca de Xira (HVFX), Hospital Santa Maria (HSM), Hospital CUF Descobertas (HCD) and Hospital Professor
Doutor Fernando Fonseca (HFF). This study was accepted by the Ethical committees of HVFX, HSM, HCD, HFF and NOVA Medical School. Participants were recruited voluntarily and written informed consent was obtained. Patients' samples were collected during clinical routine and this collection did not influence the patients' treatment or diagnosis. Sample processing was performed according to the Declaration of Helsinki.

\section{Sample processing}

Fresh tumors and biopsies were mechanically dissociated with a BD Medicon (BD Bioscience), filtered, and washed once with PBS 1X.

Low density neutrophils (LDN) and high density neutrophils (HDN) were isolated from whole blood through Histopaque-based density gradient centrifugation. Whole blood was layered on top of a solution of equal

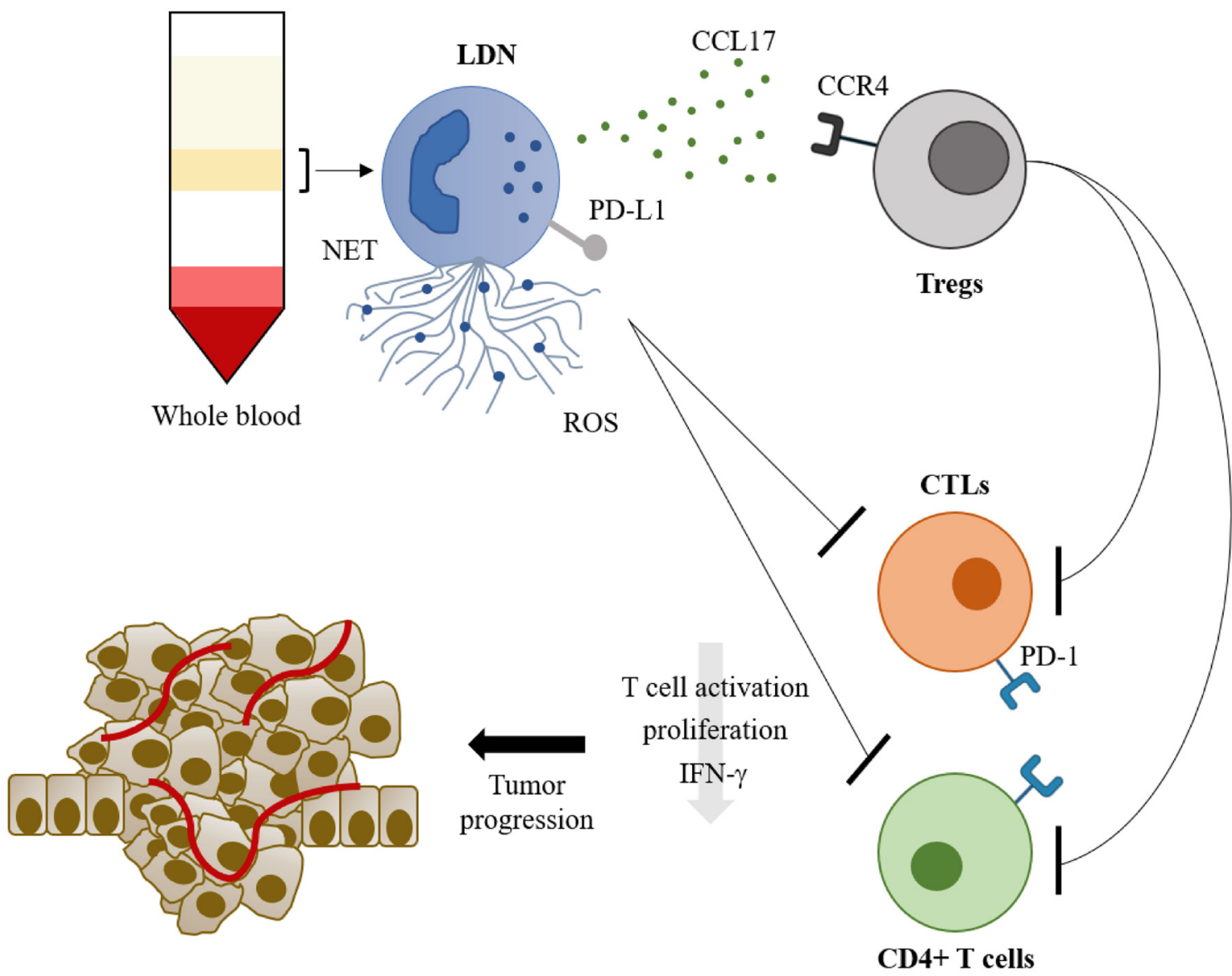

\section{No response to NACT \\ Worse prognosis}

Figure 5: The role of low density neutrophils in breast cancer prognosis and response to neoadjuvant chemotherapy. Low density neutrophils, isolated from the peripheral blood mononuclear cell layer after whole blood density centrifugation, have a high capacity to release extracellular traps (NETs) and reactive oxygen species (ROS), express PD-L1 and release CCL17. CCL17 will recruit CCR4+ regulatory $\mathrm{T}$ cells (Tregs) which will inhibit CD4+ T cells and cytotoxic CD8+ T lymphocytes (CTLs). This inhibitory action on $\mathrm{T}$ lymphocytes can also be achieved directly from the low density neutrophils. The inhibition will lead to lower T lymphocytes' activation, proliferation and IFN- $\gamma$ production, leading to tumor progression, and consequently poor response to neoadjuvant chemotherapy (NACT) and worse prognosis. 
volumes of Histopaque-1077 and Histopaque-1119 (Sigma-Aldrich), in a 1:1 (blood: Histopaque) ratio and centrifuged at $2000 \mathrm{rpm}$ for $20 \mathrm{~min}$, without brake. Circulating LDN, possibly present in the peripheral blood mononuclear cells (PBMCs) layer of the peripheral blood and HDN, present in the granulocytes fraction, were collected for immunophenotyping by flow cytometry and functional assays. The plasma was collected for ELISA assays.

\section{Immunophenotyping}

Staining for tumor-associated neutrophils was performed in processed tumor/biopsies samples with antiCD45-PercP and anti-CD15-PE (Biolegend) for $15 \mathrm{~min}$, in the dark at room temperature, followed by a washing step with PBS 1X.

Antibody staining was also performed in whole blood, and in LDN and HDN fractions. A cocktail of mouse anti-human monoclonal fluorescent antibodies (mAbs) was added to the samples and kept in the dark for $15 \mathrm{~min}$ at room temperature. For both whole blood and HDN, red blood cell lysis was performed with RBC lysis buffer (Biolegend), for $20 \mathrm{~min}$ at $4^{\circ} \mathrm{C}$, followed by a wash step with PBS $1 \mathrm{X}$ and centrifugation at $300 \mathrm{~g}$ for $5 \mathrm{~min}$.

Data were acquired in a BD FACS Canto II with FACSDiva Software v8.0.1 (BD Biosciences) and the results were analyzed using FlowJo software v10.

The mAbs used for the blood samples' staining were: anti-CD3-APC (clone UCHT1), anti-CD15-PE (clone H198), anti-CD8-PE (clone HIT8a), anti-CD4-FITC (clone OKT4), anti-CD25-PE (clone BC96), anti-CD127PE-Cy7 (clone A019D5), anti-CCR4-BV421 (L291H4), anti-CD69-PercP (clone FN50), anti-CD11b-FITC (clone ICRF44), anti-CD66b-APC (clone G10F5), anti-CD33APC-Cy7 (clone P67.6) and anti-PD-L1-APC (clone 29E.2A3), all from Biolegend. The immune populations were defined as follows: LDN (in the PBMCs fraction), HDN (in the granulocytes fraction) and total neutrophils (in whole blood) as CD15+, cytotoxic T lymphocytes as $\mathrm{CD} 3+/ \mathrm{CD} 8+$, helper $\mathrm{T}$ lymphocytes as CD3+/CD4+ and regulatory $\mathrm{T}$ cells as $\mathrm{CD} 4+/ \mathrm{CD} 25^{\text {high }} / \mathrm{CD} 127^{\text {low }}$; they are represented as a percentage in respect to the single cells' gate (see the gating strategy in Supplementary Figure 6). To analyze the expression levels of CCR4, CD11b, CD66b, CD33, PD-L1 and CD69, we considered the median fluorescent intensity (MFI) of the positive population and normalized it to the MFI of the negative population.

\section{Phagocytic capacity}

E. coli, grown in Lysogeny broth (LB) at $37^{\circ} \mathrm{C}$, were heat-killed at $95^{\circ} \mathrm{C}$ for $1 \mathrm{~h}$ and centrifuged at $12000 \mathrm{~g}$ for $10 \mathrm{~min}$. The bacterial pellet was resuspended in $0.1 \mathrm{M}$ sodium carbonate buffer $(\mathrm{pH}$ ) $)$ and incubated with $0.1 \mathrm{mg} / \mathrm{mL}$ of fluorescein isothiocyanate (FITC,
Sigma Aldrich) for $1 \mathrm{~h}$ in the dark with shaking at room temperature, followed by 3 washing steps with PBS 1X and centrifugation at $12000 \mathrm{~g}$ for $10 \mathrm{~min}$. The pellet was resuspended in PBS $1 \mathrm{X}$.

To assess phagocytic capacity, the entirety of the fraction containing the HDN or the LDN were incubated with FITC-labelled $E$. coli (1:10 bacteria to neutrophils ratio) for $30 \mathrm{~min}$ at $37^{\circ} \mathrm{C}$ or at $4^{\circ} \mathrm{C}$ (incubation without E. coli was used as negative control). Trypan blue (GE Healthcare) was added to quench FITC fluorescence of non-internalized bacteria. Cells were then washed two times with Hank's Balanced Salt Solution (HBSS, HyClone) and centrifuged at $250 \mathrm{~g}$ for $5 \mathrm{~min}$, at $8^{\circ} \mathrm{C}$. HDN were submitted to red blood cell lysis with RBC lysis buffer, as described above, followed by another washing step.

The phagocytic capacity was evaluated by flow cytometry. The internalized bacteria were estimated by measuring the ratio between the MFI of the positive population at $37^{\circ} \mathrm{C}$ and the MFI of the positive population at $4^{\circ} \mathrm{C}$, in order to discount the influence of bacteria possibly attached to the neutrophils' membrane. Higher phagocytic capacity was considered proportional to a higher value of internalized bacteria.

\section{Reactive Oxygen Species (ROS) production}

The entirety of the fraction containing the HDN or the LDN were washed 2 times with PBS $1 \mathrm{X}$ and centrifuged at $300 \mathrm{~g}$ for $5 \mathrm{~min}$. $5 \mu \mathrm{M}$ of 2',7'-Dichlorofluorescin diacetate (DCFH2-DA, Invitrogen) probe was added to each neutrophil subtype and incubated in the dark for $15 \mathrm{~min}$, at $37^{\circ} \mathrm{C}$. Following this incubation period, neutrophil stimulation was performed by adding $200 \mathrm{ng} / \mathrm{mL}$ of phorbol 12-myristate 13-acetate (PMA, Sigma Aldrich) for $30 \mathrm{~min}$. At the end of the incubation, the tubes were immediately transferred to ice to stop the stimulation and consequent release of reactive oxygen species (ROS), followed by another washing step with PBS $1 \mathrm{X}$. HDN or LDN without the probe were used as negative control, and HDN or LDN containing the probe but not the PMA stimulus were used to access the basal level of ROS production.

The oxidative burst upon stimulation was accessed by flow cytometry. The level of ROS released was determined by assessing the MFI of the stimulated neutrophils; the MFI of the non-stimulated neutrophils was also measured to assess the basal level of ROS production.

\section{Neutrophil Extracellular Traps (NETs) formation}

After patient-derived HDN and LDN isolation, red blood cell lysis was performed with RBC lysis buffer for $10 \mathrm{~min}$ at room temperature, followed by centrifugation at $1100 \mathrm{rpm}$ for $5 \mathrm{~min}$. After washing with HBSS, the 
neutrophils were resuspended in RPMI-1640 medium (Gibco) supplemented with 1\% of autologous plasma and seeded on top of coverslips (13 mm), previously soaked in $70 \%$ ethanol, in a 12-well plate. Stimulation with $100 \mathrm{ng} /$ $\mathrm{mL}$ of PMA was performed for $3 \mathrm{~h}$, at $37^{\circ} \mathrm{C}$.

After the incubation, the plate was centrifuged at $1100 \mathrm{rpm}$ for $5 \mathrm{~min}$ and the supernatant was collected, centrifuged at $2000 \mathrm{rpm}$ for $10 \mathrm{~min}$ and stored at $-20^{\circ} \mathrm{C}$ for ELISA. The wells were then washed with PBS $1 \mathrm{X}$ and fixed with $4 \%$ paraformaldehyde (PFA) for $10 \mathrm{~min}$. The wells were rinsed two times with PBT (PBS $1 \mathrm{x}+0,1 \%$ Triton X-100, ACROS Organics) and then blocked with PBT $+1 \%$ bovine serum albumin (BSA, Sigma Aldrich) for $10 \mathrm{~min}$. Mouse anti-human anti-myeloperoxidase (MPO, clone 266-6K1, Santa Cruz Biotechnology) was added in a 1:100 concentration and incubated for $1 \mathrm{~h}$ at room temperature. The secondary antibody goat anti-mouse Alexa 568 (Invitrogen) was added at a concentration of 1:500 and incubated for $45 \mathrm{~min}$ in the dark at room temperature. Counterstaining was performed with 4',6-diamidino-2-phenylindole (DAPI) solution $(0.001 \mathrm{mg} / \mathrm{mL}$ in $\mathrm{PBS} 1 \mathrm{X})$, for $10 \mathrm{~min}$ protected from light, and the coverslips were mounted with Fluorescent Mounting Media (DAKO) into microscopy slides. Images were acquired in a confocal microscope (LSM710, Zeiss) and analyzed with Fiji software.

Nuclei area was assessed in the DAPI channel by applying an automatic threshold and measuring the area in the "analyze particles" menu. NETs' area was quantified also by applying an automatic threshold. Besides the area, NETs were assessed by measuring the fluorescence intensity of MPO in the Alexa 568 channel. In all quantifications, 3 different images per patient were analyzed and the mean value was obtained.

\section{Co-culture of LDN with PBMCs}

Whole blood from 10 breast cancer patients was collected and the PBMCs fraction was isolated as described above. PBMCs were stained with anti-CD15$\mathrm{PE}$ for $15 \mathrm{~min}$ in the dark at $4^{\circ} \mathrm{C}$, followed by a washing step with PBS $1 X$ and centrifugation at $300 \mathrm{~g}, 5 \mathrm{~min}$. Cells were resuspended in PBS with $2 \%$ fetal bovine serum (FBS, Sigma Aldrich) and 10\% Penicillin/Streptomycin (GE Healthcare). CD15+ (LDN) and CD15-depleted (PBMCs) populations from the same patient were sorted in the BD FACS Aria III and collected to RPMI-1640 with $10 \%$ FBS and 10\% Penicillin/Streptomycin. CD15depleted population was cultured alone or in a $1: 1$ ratio with CD15+ cells in RPMI-1640 with $10 \%$ FBS and 1\% Penicillin/Streptomycin. Stimulation was performed with $35 \mathrm{ng} / \mathrm{mL}$ of PMA and $1 \mu \mathrm{g} / \mathrm{mL}$ of ionomycin (Merck Millipore) for $24 \mathrm{~h}$. After this incubation period, the supernatant was collected as above described and the cells were stained with anti-CD3-PercP (clone UCHT1), antiCD4 (clone OKT4), anti-CD8-PacificBlue (clone SK1),
anti-CD25 (clone BC96), anti-HLA-DR-APC (clone L243), anti-CD69-APC-Cy7 (clone FN50) and anti-Ki67$\mathrm{PE}$ (clone Ki-67). The staining was executed as described above, except for the intracellular marker Ki67, which was added only after a 30 min permeabilization step with Fix/Perm kit (Invitrogen). Incubation with Ki67 was performed for $30 \mathrm{~min}$ at room temperature, followed by a wash step with PBS $1 \mathrm{X}$ and centrifugation at $300 \mathrm{~g}$ for $5 \mathrm{~min}$.

\section{ELISA}

The quantity of secreted IL-8, IL-10, IL-17, IFN- $\gamma$, TGF- $\beta$ (Biolegend) and CCL17 (R\&D Systems) in the patients' plasma was measured using the ELISA technique, according to the manufacturer's instructions. CCL17 and IFN- $\gamma$ were also measured in the culture's supernatant. Cytokine concentration was calculated using the specific standard curves.

\section{Statistical analysis}

Statistical analysis was performed in GraphPad Prism v8 and statistical significance was considered for $p<0.05$. Comparison between samples was performed by a nonparametric Mann-Whitney test or by a two-way ANOVA with multiple comparisons. Correlations were calculated with the Spearman $r$ test. $T$-test was used to compare samples in an unstimulated $v s$ stimulated condition. Progression-free survival was plotted as a Kaplan-Meier curve and the log-rank test was used to assess the hazard ratios.

\section{Abbreviations}

BC: Breast Cancer; CTLs: cytotoxic T lymphocytes; ER: estrogen receptor; HDN: High density neutrophils; HER2: human epidermal growth factor receptor 2; LDN: Low density neutrophils; MFI: median fluorescence intensity; MPO: myeloperoxidase; NACT: neoadjuvant chemotherapy; NETs: neutrophil extracellular traps; NLR: Neutrophil-to-lymphocyte ratio; PBMCs: Peripheral blood mononuclear cells; pCR: pathological complete response; PD-L1: programmed cell death ligand 1; PR: progesterone receptor; ROS: reactive oxygen species; TAN: Tumor-associated neutrophils; TIME: Tumor immune microenvironment; TNBC: Triple Negative Breast Cancer; Tregs: regulatory T cells.

\section{Author contributions}

DPS and BFC contributed equally to this work. Both conducted all the experiments, analyzed, and interpreted the data, performed the statistical analysis, assembled all the figures and wrote the manuscript. RS and NS helped in the execution of flow cytometry experiments. AJ contributed 
to scientific discussion. SB helped in the obtainment of patients' samples and clinical data. MGC designed and supervised the study, interpreted the data and wrote the manuscript. All the authors approved the final manuscript.

\section{ACKNOWLEDGMENTS}

We would like to thank all breast cancer patients that agreed to participate in this study. We also appreciate all the help from Nurses, Oncologists and Study Coordinators of Hospital de Vila Franca de Xira (HVFX), Hospital Professor Doutor Fernando Fonseca (HFF) and Centro de Investigação Clinica of Hospital de Santa Maria (HSM) that helped to obtain blood samples. We also acknowledge the help from Radiologists, Surgeons and Oncologists of Hospital CUF Descobertas, HVFX and HFF which assisted in the obtainment of fresh biopsies and tumor samples. We would also like to thank the Flow Cytometry Facility of CEDOC.

\section{CONFLICTS OF INTEREST}

The authors have declared no conflicts of interest.

\section{FUNDING}

This work was supported by Liga Portuguesa Contra o Cancro; Fundação para a Ciência e Tecnologia (PD/BD/114023/2015, SFRH/BD/148422/2019 and 2021.08031.BD); and iNOVA4Health (UIDB/04462/2020 and DAI/2019/46).

\section{REFERENCES}

1. Bray F, Ferlay J, Soerjomataram I, Siegel RL, Torre LA, Jemal A. Global cancer statistics 2018: GLOBOCAN estimates of incidence and mortality worldwide for 36 cancers in 185 countries. CA Cancer J Clin. 2018; 68:394 424. https://doi.org/10.3322/caac.21492. [ [PubMed]

2. Pruneri G, Vingiani A, Denkert C. Tumor infiltrating lymphocytes in early breast cancer. Breast. 2018; 37:20714. https://doi.org/10.1016/j.breast.2017.03.010. [PubMed]

3. Li X, Dai D, Chen B, Tang H, Xie X, Wei W. The value of neutrophil-to-lymphocyte ratio for response and prognostic effect of neoadjuvant chemotherapy in solid tumors: A systematic review and meta-analysis. J Cancer. 2018; 9:861-71. https://doi.org/10.7150/jca.23367. [PubMed]

4. Ivars Rubio A, Yufera JC, de la Morena P, Fernández Sánchez A, Navarro Manzano E, García Garre E, García Martinez E, Marín Zafra G, Sánchez Cánovas M, García Torralba E, Ayala de la Peña F. Neutrophil-lymphocyte ratio in metastatic breast cancer is not an independent predictor of survival, but depends on other variables. Sci Rep. 2019; 9:16979. https:// doi.org/10.1038/s41598-019-53606-3. [PubMed]
5. Vano YA, Oudard S, By MA, Têtu P, Thibault C, Aboudagga H, Scotté F, Elaidi R. Optimal cut-off for neutrophilto-lymphocyte ratio: Fact or Fantasy? A prospective cohort study in metastatic cancer patients. PLoS One. 2018; 13:e0195042. https://doi.org/10.1371/journal. pone.0195042. [PubMed]

6. Beatty GL, Gladney WL. Immune escape mechanisms as a guide for cancer immunotherapy. Clin Cancer Res. 2015; 21:687-92. https://doi.org/10.1158/1078-0432.CCR-141860. [PubMed]

7. Fridlender ZG, Sun J, Kim S, Kapoor V, Cheng G, Ling L, Worthen GS, Albelda SM. Polarization of tumor-associated neutrophil phenotype by TGF-beta: "N1" versus "N2" TAN. Cancer Cell. 2009; 16:183-94. https://doi.org/10.1016/j. ccr.2009.06.017. [PubMed]

8. Shaul ME, Levy L, Sun J, Mishalian I, Singhal S, Kapoor V, Horng W, Fridlender G, Albelda SM, Fridlender ZG. Tumor-associated neutrophils display a distinct N1 profile following TGF $\beta$ modulation: A transcriptomics analysis of pro- vs. antitumor TANs. Oncoimmunology. 2016; 5:e1232221. https://doi.org/10.1080/216240 2X.2016.1232221. [PubMed]

9. Cloke T, Munder M, Taylor G, Müller I, Kropf P. Characterization of a novel population of low-density granulocytes associated with disease severity in HIV1 infection. PLoS One. 2012; 7:e48939. https://doi. org/10.1371/journal.pone.0048939. [PubMed]

10. Fu J, Tobin MC, Thomas LL. Neutrophil-like low-density granulocytes are elevated in patients with moderate to severe persistent asthma. Ann Allergy Asthma Immunol. 2014; 113:635-40.e2. https://doi.org/10.1016/j. anai.2014.08.024. [PubMed]

11. Liu Y, Hu Y, Gu F, Liang J, Zeng Y, Hong X, Zhang K, Liu L. Phenotypic and clinical characterization of low density neutrophils in patients with advanced lung adenocarcinoma. Oncotarget. 2017; 8:90969-78. https://doi.org/10.18632/ oncotarget.18771. [PubMed]

12. Rakic A, Beaudry P, Mahoney DJ. The complex interplay between neutrophils and cancer. Cell Tissue Res. 2018; 371:517-29. https://doi.org/10.1007/s00441-017-2777-7. [PubMed]

13. Sagiv JY, Michaeli J, Assi S, Mishalian I, Kisos H, Levy L, Damti P, Lumbroso D, Polyansky L, Sionov RV, Ariel A, Hovav AH, Henke E, et al. Phenotypic diversity and plasticity in circulating neutrophil subpopulations in cancer. Cell Rep. 2015; 10:562-73. https://doi.org/10.1016/j. celrep.2014.12.039. [PubMed]

14. Michaeli J, Shaul ME, Mishalian I, Hovav AH, Levy L, Zolotriov L, Granot Z, Fridlender ZG. Tumor-associated neutrophils induce apoptosis of non-activated CD8 T-cells in a TNF $\alpha$ and NO-dependent mechanism, promoting a tumor-supportive environment. Oncoimmunology. 2017; 6:e1356965. https://doi.org/10.1080/216240 2X.2017.1356965. [PubMed] 
15. Wang TT, Zhao YL, Peng LS, Chen N, Chen W, Lv YP, Mao FY, Zhang JY, Cheng P, Teng YS, Fu XL, Yu PW, Guo $\mathrm{G}$, et al. Tumour-activated neutrophils in gastric cancer foster immune suppression and disease progression through GM-CSF-PD-L1 pathway. Gut. 2017; 66:1900-11. https:// doi.org/10.1136/gutjnl-2016-313075. [PubMed]

16. Hsu BE, Tabariès S, Johnson RM, Andrzejewski $\mathrm{S}$, Senecal J, Lehuédé C, Annis MG, Ma EH, Völs S, Ramsay L, Froment R, Monast A, Watson IR, et al. Immature Low-Density Neutrophils Exhibit Metabolic Flexibility that Facilitates Breast Cancer Liver Metastasis. Cell Rep. 2019; 27:3902-15.e6. https://doi.org/10.1016/j. celrep.2019.05.091. [PubMed]

17. Park J, Wysocki RW, Amoozgar Z, Maiorino L, Fein MR, Jorns J, Schott AF, Kinugasa-Katayama Y, Lee Y, Won NH, Nakasone ES, Hearn SA, Küttner V, et al. Cancer cells induce metastasis-supporting neutrophil extracellular DNA traps. Sci Transl Med. 2016; 8:361ra138. https://doi. org/10.1126/scitranslmed.aag1711. [PubMed]

18. Chollet P, Amat S, Cure H, de Latour M, Le Bouedec G, Mouret-Reynier MA, Ferriere JP, Achard JL, Dauplat J, Penault-Llorca F. Prognostic significance of a complete pathological response after induction chemotherapy in operable breast cancer. Br J Cancer. 2002; 86:1041-46. https://doi.org/10.1038/sj.bjc.6600210. [PubMed]

19. Resende U, Cabello C, Ramalho SOB, Zeferino LC. Prognostic assessment of breast carcinoma submitted to neoadjuvant chemotherapy with pathological noncomplete response. BMC Cancer. 2019; 19:601. https://doi. org/10.1186/s12885-019-5812-0. [PubMed]

20. Saraiva DP, Jacinto A, Borralho P, Braga S, Cabral MG. HLA-DR in Cytotoxic T Lymphocytes Predicts Breast Cancer Patients' Response to Neoadjuvant Chemotherapy. Front Immunol. 2018; 9:2605. https://doi.org/10.3389/ fimmu.2018.02605. [PubMed]

21. Chen Y, Chen K, Xiao X, Nie Y, Qu S, Gong C, Su F, Song E. Pretreatment neutrophil-to-lymphocyte ratio is correlated with response to neoadjuvant chemotherapy as an independent prognostic indicator in breast cancer patients: a retrospective study. BMC Cancer. 2016; 16:320. https://doi. org/10.1186/s12885-016-2352-8. [PubMed]

22. Bae SJ, Cha YJ, Yoon C, Kim D, Lee J, Park S, Cha C, Kim JY, Ahn SG, Park HS, Park S, Kim SI, Jeong J. Prognostic value of neutrophil-to-lymphocyte ratio in human epidermal growth factor receptor 2-negative breast cancer patients who received neoadjuvant chemotherapy. Sci Rep. 2020; 10:13078. https://doi.org/10.1038/s41598-020-69965-1. [PubMed]

23. Kolaczkowska E, Kubes P. Neutrophil recruitment and function in health and inflammation. Nat Rev Immunol. 2013; 13:159-75. https://doi.org/10.1038/nri3399. [PubMed]

24. Cools-Lartigue J, Spicer J, McDonald B, Gowing S, Chow S, Giannias B, Bourdeau F, Kubes P, Ferri L. Neutrophil extracellular traps sequester circulating tumor cells and promote metastasis. J Clin Invest. 2013; 123:3446-58. https://doi.org/10.1172/JCI67484. [PubMed]

25. Najmeh S, Cools-Lartigue J, Rayes RF, Gowing S, Vourtzoumis P, Bourdeau F, Giannias B, Berube J, Rousseau S, Ferri LE, Spicer JD. Neutrophil extracellular traps sequester circulating tumor cells via $\beta 1$-integrin mediated interactions. Int J Cancer. 2017; 140:2321-30. https://doi. org/10.1002/ijc.30635. [PubMed]

26. Wu L, Saxena S, Awaji M, Singh RK. Tumor-Associated Neutrophils in Cancer: Going Pro. Cancers (Basel). 2019; 11:564. https://doi.org/10.3390/cancers11040564. [PubMed]

27. Mishalian I, Bayuh R, Eruslanov E, Michaeli J, Levy L, Zolotarov L, Singhal S, Albelda SM, Granot Z, Fridlender ZG. Neutrophils recruit regulatory T-cells into tumors via secretion of CCL17--a new mechanism of impaired antitumor immunity. Int J Cancer. 2014; 135:1178-86. https://doi.org/10.1002/ijc.28770. [PubMed]

28. Shen M, Hu P, Donskov F, Wang G, Liu Q, Du J. Tumorassociated neutrophils as a new prognostic factor in cancer: a systematic review and meta-analysis. PLoS One. 2014; 9:e98259. https://doi.org/10.1371/journal.pone.0098259. [PubMed]

29. Wang X, Qiu L, Li Z, Wang XY, Yi H. Understanding the Multifaceted Role of Neutrophils in Cancer and Autoimmune Diseases. Front Immunol. 2018; 9:2456. https://doi.org/10.3389/fimmu.2018.02456. [PubMed]

30. Coffelt SB, Wellenstein MD, de Visser KE. Neutrophils in cancer: neutral no more. Nat Rev Cancer. 2016; 16:431-46. https://doi.org/10.1038/nrc.2016.52. [PubMed]

31. Tabariès S, Ouellet V, Hsu BE, Annis MG, Rose AA, Meunier L, Carmona E, Tam CE, Mes-Masson AM, Siegel PM. Granulocytic immune infiltrates are essential for the efficient formation of breast cancer liver metastases. Breast Cancer Res. 2015; 17:45. https://doi.org/10.1186/s13058015-0558-3. [PubMed]

32. Saraiva DP, Guadalupe Cabral M, Jacinto A, Braga S. How many diseases is triple negative breast cancer: the protagonism of the immune microenvironment. ESMO Open. 2017; 2:e000208. https://doi.org/10.1136/ esmoopen-2017-000208. [PubMed]

33. Faria SS, Fernandes PC Jr, Silva MJ, Lima VC, Fontes W, Freitas-Junior R, Eterovic AK, Forget P. The neutrophil-to-lymphocyte ratio: a narrative review. Ecancermedicalscience. 2016; 10:702. https://doi. org/10.3332/ecancer.2016.702. [PubMed]

34. Bowers NL, Helton ES, Huijbregts RP, Goepfert PA, Heath SL, Hel Z. Immune suppression by neutrophils in HIV-1 infection: role of PD-L1/PD-1 pathway. PLoS Pathog. 2014; 10:e1003993. https://doi.org/10.1371/journal.ppat.1003993. [PubMed]

35. Salmaninejad A, Valilou SF, Shabgah AG, Aslani S, Alimardani M, Pasdar A, Sahebkar A. PD-1/PD-L1 pathway: Basic biology and role in cancer immunotherapy. $\mathrm{J}$ 
Cell Physiol. 2019; 234:16824-37. https://doi.org/10.1002/ jcp.28358. [PubMed]

36. Aggarwal V, Tuli HS, Varol A, Thakral F, Yerer MB, Sak K, Varol M, Jain A, Khan MA, Sethi G. Role of Reactive Oxygen Species in Cancer Progression: Molecular Mechanisms and Recent Advancements. Biomolecules. 2019; 9:735. https://doi.org/10.3390/biom9110735. [PubMed]

37. Singel KL, Segal BH. Neutrophils in the tumor microenvironment: trying to heal the wound that cannot heal. Immunol Rev. 2016; 273:329-43. https://doi. org/10.1111/imr.12459. [PubMed]

38. Papayannopoulos V, Metzler KD, Hakkim A, Zychlinsky A. Neutrophil elastase and myeloperoxidase regulate the formation of neutrophil extracellular traps. J Cell Biol. 2010; 191:677-91. https://doi.org/10.1083/jcb.201006052. [PubMed]

39. Németh T, Mócsai A, Lowell CA. Neutrophils in animal models of autoimmune disease. Semin Immunol. 2016; 28:174-86. https://doi.org/10.1016/j.smim.2016.04.001. [PubMed]
40. Odobasic D, Kitching AR, Yang Y, O'Sullivan KM, Muljadi RC, Edgtton KL, Tan DS, Summers SA, Morand EF, Holdsworth SR. Neutrophil myeloperoxidase regulates T-cell-driven tissue inflammation in mice by inhibiting dendritic cell function. Blood. 2013; 121:4195-204. https:// doi.org/10.1182/blood-2012-09-456483. [PubMed]

41. Hao Y, Baker D, Ten Dijke P. TGF- $\beta$-Mediated EpithelialMesenchymal Transition and Cancer Metastasis. Int J Mol Sci. 2019; 20:2767. https://doi.org/10.3390/ijms20112767. [PubMed]

42. Derynck R, Akhurst RJ, Balmain A. TGF-beta signaling in tumor suppression and cancer progression. Nat Genet. 2001; 29:117-29. https://doi.org/10.1038/ng1001-117. [PubMed]

43. Flavell RA, Sanjabi S, Wrzesinski SH, Licona-Limón P. The polarization of immune cells in the tumour environment by TGFbeta. Nat Rev Immunol. 2010; 10:554-67. https://doi. org/10.1038/nri2808. [PubMed]

44. Granot Z. Neutrophils as a Therapeutic Target in Cancer. Front Immunol. 2019; 10:1710. https://doi.org/10.3389/ fimmu.2019.01710. [PubMed] 